\title{
Reversible Alcohol-related Dementia: A Five-year Follow-up Study Using FDG-PET and Neuropsychological Tests
}

\author{
Tomohiko Asada ${ }^{1}$, Shigetoshi Takaya ${ }^{1,2}$, Yoshihiro Takayama ${ }^{3}$, Hiroshi Yamauchi ${ }^{1}$, \\ Kazuo Hashikawa ${ }^{1,4}$ and Hidenao Fukuyama ${ }^{1}$
}

\begin{abstract}
Objective As the pathophysiology of alcohol-related dementia (ARD) is unclear, we examined a patient with reversible ARD using neuropsychological tests and ${ }^{18} \mathrm{~F}$-fluorodeoxyglucose positron emission tomography (FDG-PET).

Methods Design: A five-year follow-up case study with neuropsychological tests and FDG-PET. Setting: Kyoto University Hospital.

Patients A 42-year-old patient who was unable to perform his office duties because of slowly progressive amnesia with executive dysfunction.

Results The initial evaluation with neuropsychological tests showed severe verbal memory disturbance. The patient did not discuss his excessive alcohol consumption in the initial history-taking session and thiamine deficiency was absent; therefore, early-stage Alzheimer's disease was suspected. Later, the patient revealed prior excessive alcohol intake and his cognitive function improved markedly after a period of abstinence. Retrospective analysis of initial FDG-PET images using a voxel-wise statistical method revealed glucose hypometabolism in the diencephalon and basal forebrain. Follow-up for 5 years after the initial evaluation showed improved cognitive function and recovery of glucose metabolism in the two brain regions.

Conclusion Hypofunction in the diencephalon and basal forebrain was associated with cognitive decline in our patient. This case may provide evidence for the etiopathic brain regions in reversible type ARD.
\end{abstract}

Key words: alchohol related dementia, Wernicke-Korsakoff syndrome, FDG-PET, Alzheimer disease, diencephalon, basal forebrain

(Inter Med 49: 283-287, 2010)

(DOI: 10.2169/internalmedicine.49.2662)

\section{Introduction}

The Wernicke-Korsakoff syndrome (WKS) is a representative alcohol-induced memory disturbance characterized by neuropathological changes in the diencephalon, including the anterior part of the thalamus, and the mammillary body caused by thiamine deficiency (1). The most characteristic neuropsychological feature of WKS is marked memory deficits, while the other intellectual abilities are relatively preserved $(2,3)$. An ${ }^{18} \mathrm{~F}$-fluorodeoxyglucose positron emission tomography (FDG-PET) study of WKS showed regional glucose hypometabolism in the diencephalic grey matter, which is consistent with the underlying neuropathology. Glucose hypometabolism was also found in the medial temporal lobe and retrosplenium, which is interpreted as secondary metabolic effects within the diencephalic-limbic memory circuits (4).

Alcohol-related dementia (ARD) has been broadly defined as alcohol-induced dementia in the Diagnostic and Statistical Manual of Mental Disorders IV- Text Revision (DSM-IVTR). ARD has been reported as the organic brain syndrome induced by alcohol abuse, which results in severe cognitive impairment, including executive dysfunction and lack of

\footnotetext{
${ }^{1}$ Human Brain Research Center, Kyoto University Graduate School of Medicine, Kyoto, ${ }^{2}$ Radioisotope Research Center, Kyoto University, Kyoto, ${ }^{3}$ Department of Speech Physiology, Graduate School of Medicine, University of Tokyo, Tokyo and ${ }^{4}$ National Hospital Organization, Osaka Minami Medical Center, Kawachinagano

Received for publication July 7, 2009; Accepted for publication October 8, 2009

Correspondence to Dr. Hidenao Fukuyama, fukuyama@kuhp.kyoto-u.ac.jp
} 
Table 1. Results of Neuropsychological Assessments

\begin{tabular}{llll}
\hline Battery & On admission & $\begin{array}{l}3 \text { weeks } \\
\text { after admission }\end{array}$ & $\begin{array}{l}5 \text { years } \\
\text { after discharge }\end{array}$ \\
\hline $\begin{array}{l}\text { Mini-Mental State Examination } \\
\text { WAIS-R }\end{array}$ & 30 (full marks) & ND & 30 \\
$\quad$ VIQ & 100 & ND & 130 \\
$\quad$ PIQ & 107 & ND & 137 \\
$\quad$ FIQ & 104 & ND & 137 \\
Geriatric Depression Scale & 15 (full marks) & ND & 0 \\
Miyake's Verbal Memory Test & & & \\
$\quad$ Associated paired-word test & $9-10-10$ & ND & $10-10-10$ \\
$\quad$ Nonassociated paired-word test & $1-4-4$ & $6-7-6$ & $8-10-10$ \\
\hline
\end{tabular}

WAIS-R: Wechsler Adult Intelligence Scale-Revised ; VIQ: verbal intelligence quotient;

PIQ: performance intelligence quotient; FIQ: full-scale intelligence quotient; ND: not done

emotional control, in addition to memory disturbance (5). Although the pathophysiology of WKS has been relatively well studied, the neural basis of ARD remains unclear. Two concepts have been proposed for ARD: 1) ARD is a variant of WKS and is associated with thiamine deficiency-induced dysfunction in the diencephalon (6) and 2) ARD originates from the disturbance impairment of the cholinergic system, including projection fibers from the nucleus basalis of Meynert or the dorsal brainstem (7-12). Functional neuroimaging techniques such as PET have been expected to elucidate the neural substrate of ARD (13).

Here, we describe a series of neuropsychological and FDG-PET studies over 5 years for a 42-year-old male office worker with dementia resulting from excessive alcohol intake for several years. To the best of our knowledge, there has been no report showing that regional cerebral glucose hypometabolism improves with the amelioration of neuropsychological impairment in a patient with ARD. We discuss the possible neural basis of reversible ARD.

\section{Case Report}

A 42-year-old man was admitted to hospital for the evaluation of his slowly progressive impairment in recent memory. He reported that he had been suffering from daily stress at work and forgetfulness for a few years. He complained that he had difficulty in concentrating and thinking and lacked motivation, although he did not report appetite loss or sleep disturbance. For 6 months before admission, his supervisor had recognized that his memory disturbance was becoming worse. Although he made notes to remember some things, he often forgot to write them down. He lost his way home once. He often lost his temper, but sometimes could not remember the reason for his behavior. He gradually became unable to do his work. He had no history of neurological or psychiatric illness. Since he had not revealed a history of excessive alcohol intake, an early stage of Alzheimer's disease was initially suspected, and he was admitted to the hospital for the evaluation of dementia.

On admission, he showed mild cognitive decline and im- pairment in emotional control and concentration. He appeared to be apathic. In his general and neurological examination, extraocular movements were full and truncal or limb ataxia was not noted. He was slightly disoriented, and his recent memory was definitely disturbed, while his remote memory was relatively preserved. Neuropsychological batteries on admission showed an immediate verbal memory decline. The geriatric depression scale (GDS) revealed the worst possible score (15 points), which indicated that he was in a depressive state (see Neuropsychological Assessment in Results). Blood tests revealed a slight elevation of the levels of aspartate and alanine aminotransferases and triglycerides, thereby suggesting fatty liver. Serum thiamine level $(37 \mathrm{ng} / \mathrm{mL})$ was within the normal range $(20-50 \mathrm{ng} /$ $\mathrm{mL}$ ), while serum vitamin $B_{12}$ level was $323 \mathrm{pg} / \mathrm{mL}$ (normal range, 249-938 $\mathrm{pg} / \mathrm{mL}$ ). Serological tests for syphilis were negative, and thyroid function was normal. The finding of brain magnetic resonance imaging was unremarkable. Electroencephalography showed generalized intermittent irregular slow waves (theta range). His memory and emotional control improved gradually after admission to hospital. The score of Miyake's Verbal Memory Test improved at 3 weeks after admission as compared to those performed on admission (Table 1). Since his motivation did not improve despite the recovery of memory function, a low dose of an antidepressant (25 mg/day of maprotiline hydrochloride) was started.

One year after discharge, he confessed that he had a history of daily excessive alcohol intake for several years prior to admission. He had consumed 2 drinks/day for several years and 6 drinks/day for 1 year prior to admission (1 drink equals $12 \mathrm{~g}$ of pure ethanol; National Institute on Alcohol Abuse and Alcoholism recommends alcohol consumption of $\leq 2$ drinks/day for people of similar age) $(1,14)$. He had abstained from alcohol since admission to hospital, although he had shown no symptoms of withdrawal during hospitalization. This information confirmed the final diagnosis as a reversible type of ARD. To elucidate the pathophysiology of this unique condition, we conducted a follow-up study with FDG-PET and neuropsychological tests for 5 
years after hospitalization. At present, the patient is working in a middle management position with no problems at work.

\section{Methods}

\section{Neuropsychological tests}

The Japanese edition of the Wechsler Adult Intelligence Scale-Revised (WAIS-R) was used for the assessment of intelligence. Miyake's Paired-associated Word Learning Test for Verbal Memory was used as the verbal memory test. In this test, the subject is requested to memorize 10 pairs of either associated or non-associated nouns and immediately recollect the paired word. This procedure is repeated 3 times with a time interval. The results are expressed as the number of words recalled accurately in each procedure $(15,16)$. The Japanese version of the Geriatric Depression Scale (GDS) was used as the depression scale, although the patient's age was younger than the target age group considered for this scale (17). The Mini-Mental State Examination (MMSE) was used for a simple intelligence scale.

\section{Image data acquisition and analyses}

FDG-PET was performed using a General Electric Advance PET scanner (General Electric Medical Systems, Milwaukee, WI, USA). A $370 \mathrm{MBq}(10 \mathrm{mCi})$ of $\left[{ }^{18} \mathrm{~F}\right]-\mathrm{FDG}$ was injected intravenously into the patient. Forty minutes after administration of the radiotracer, 35 slices of brain emission images were acquired over a 20 -min period. The emission images were reconstructed using an iterative reconstruction method. All reconstructed images were corrected for attenuation by using ${ }^{68} \mathrm{Ge}-{ }^{68} \mathrm{Ga}$ transmission scans.

In voxel-wise analyses using SPM2 software (Wellcome Department of Imaging Neuroscience, UCL, London, UK), spatially normalized images were smoothed with an isotopic Gaussian Kernel set at 16-mm full-width at half-maximum (FWHM). To remove the effect of the global count, the count of each voxel was normalized to the total count of the brain by using proportional scaling. The FDG-PET image of the patient was compared with those of 12 age-matched controls (mean age, $37.3 \pm 12.9$ years) by using $t$ statistics. We investigated hypometabolic brain areas for the patient as compared with control subjects. The regions were considered to be significant at a height threshold of $\mathrm{p}=0.01$, uncorrected for multiple comparison $(\mathrm{Z}$ score $=2.34)$, and an extent threshold of 50 voxels. For visualization, significant clusters were projected onto a surface-rendered anatomical template provided by SPM2. The spatial coordinates of the local maxima from the statistical analysis were used to identify the corresponding brain areas according to the atlas of Talairach and Tournoux (18). The nonlinear transformation of the Montreal Neurological Institute (MNI) coordinates into the Talairach coordinates was performed using appropriate converter software (mni2tal.m; http://www.mrc-cbu.cam. ac.uk/Imaging/Common/mnispace.shtml).

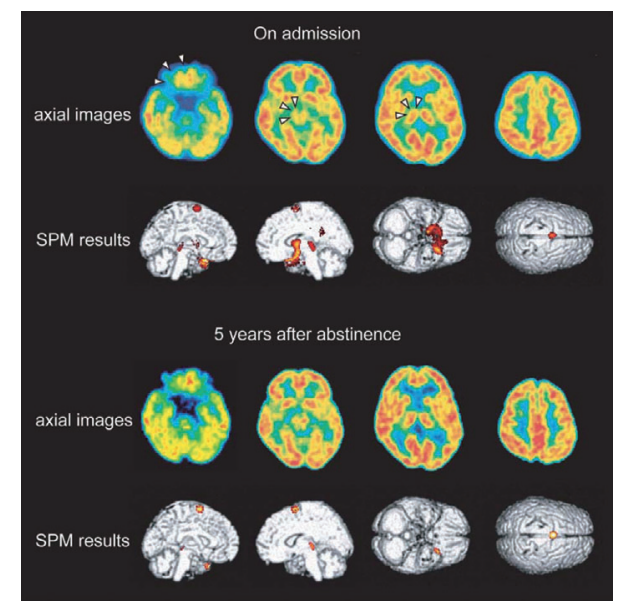

Figure 1. This figure shows axial images of FDG-PET and the results of the voxel-wise statistical comparison with healthy subjects $(n=12)$ by using SPM2 (height threshold, $\mathrm{z}>\mathbf{2 . 3 4}, \mathrm{p}<\mathbf{0 . 0 1}$; extent threshold $>50$ voxels). FDG-PET on admission showed glucose hypometabolism in the right diencephalon, including the anterior thalamus and the bilateral basal forebrains more on the right side (white arrow heads). The SPM results clarified that cerebral glucose hypometabolism improved at 5 years after abstinence in the right diencephalon and bilateral basal forebrains and temporal poles.

\section{Results}

\section{Neuropsychological assessments}

As shown in Table 1, a trend of amelioration was found in all neuropsychological batteries. Among these batteries, the score of the nonassociated paired-word test in Miyake's Verbal Memory Test was markedly improved at 5 years after discharge. The depression scale score at 5 years after discharge also showed complete amelioration as compared to that at admission.

\section{FDG-PET analyses by SPM2}

As shown in Fig. 1 and Table 2, the voxel-based statistical analysis using SPM2 for the first FDG-PET revealed glucose hypometabolism in the right diencephalon, including the anterior thalamus; the bilateral basal forebrains, temporal poles and supplementary motor areas (SMAs); the dorsal brainstem. The subsequent FDG-PET analysis at 5 years after discharge revealed improvement in cerebral glucose metabolism in the right diencephalon and the bilateral basal forebrains and temporal poles.

Although glucose hypometabolism was still detected to some extent in the temporal pole, SMA and dorsal basal brain stem, magnetic resonance imaging showed no anatomical abnormalities in such regions. 
Table 2. Brain Regions of Our Patient Showing a Significant Decrease in Glucose Metabolism in FDG-PET Study on Admission and 5 Years after Abstinence as Compared with Those of the Healthy Subjects

\begin{tabular}{|c|c|c|c|c|c|}
\hline \multirow[t]{2}{*}{ Brain region } & \multirow[t]{2}{*}{ Side } & \multicolumn{3}{|c|}{ Talairach coordinates of the peak } & \multirow[b]{2}{*}{ Z score } \\
\hline & & $\mathrm{x}$ & $\mathrm{y}$ & $\mathrm{z}$ & \\
\hline \multicolumn{6}{|l|}{ On admission } \\
\hline Diencephalon & $\mathrm{R}$ & 12 & -2 & 6 & 3.94 \\
\hline Temporal pole & $\mathrm{L}$ & -24 & 18 & -29 & 3.91 \\
\hline Orbitofrontal gyrus & $\mathrm{R}$ & 10 & 10 & -24 & 3.19 \\
\hline Retrosplenium & $\mathrm{R}$ & 32 & -44 & 21 & 3.34 \\
\hline SMA & $\mathrm{L}$ & -2 & 3 & 64 & 2.85 \\
\hline Dorsal brain stem & - & 8 & -27 & 5 & 2.84 \\
\hline \multicolumn{6}{|c|}{5 years after abstinence } \\
\hline Temporal pole & $\mathrm{L}$ & -24 & 18 & -29 & 3.14 \\
\hline SMA & $\mathrm{L}$ & 8 & -27 & 5 & 2.93 \\
\hline Dorsal brain stem & - & 8 & -27 & 5 & 2.66 \\
\hline
\end{tabular}

R: right; L: left, SMA: supplementary motor cortex

\section{Discussion}

\section{Etiopathic brain regions for tentative cognitive de- cline}

The patient had no evidence of alcohol dependency throughout the clinical course, although the patient had a history of excessive alcohol drinking. We consider the patient did not have acute abstinent syndrome, which was originates from not only alcohol but also from drugs, because our patient did not have abstinent syndrome during the hospitalization, and the patient's MMSE score was full. We also consider that acute brain syndrome, like black out, due to excessive alcohol drinking, or caused by repetitive toxicity, might was not seen in the patient, throughout this patient's hospitalization.

In the present patient, cerebral glucose hypometabolism was initially detected in the diencephalon and basal forebrain. Five years of abstinence resulted in improvement in cerebral glucose metabolism in these regions and cognitive function. This might suggest that the tentative hypofunction in these 2 brain regions was ascribed to the reversible nature of cognitive dysfunction in our patient.

The diencephalon and basal forebrain have been associated with amnesic diseases such as WKS or Alzheimer's disease. In WKS, neuropathological changes occur in the memory circuit in the diencephalons such as the anterior nuclei of the thalami, the mammillary bodies, and mammillothalamic tracts (19-22). A more recent FDG-PET study revealed that glucose metabolism is decreased in these brain regions (4). Thus, both the structural and functional changes in the diencephalons are ascribed to the irreversible nature of cognitive decline in WKS (1).

Cognitive impairment in WKS is also ascribed to the cholinergic pathway from the basal forebrain, as in the case of Alzheimer's disease (23). Thiamine is thought to play a role in the cholinergic synaptic transmission and axonal conduction, and an excessive alcohol intake can result in thiamine deficiency (5). In our patient, the cholinergic neurons in the basal forebrain might be impaired tentatively because of either direct toxicity of excessive alcohol or a rapid decrease in thiamine concentration in the blood, although the thiamine concentration was within the normal range.

Although ARD might represent a heterogeneous disease concept, including a variant of WKS, the present case might provide a clue to elucidate the etiopathic brain regions in the reversible type of ARD. It remains to be studied whether the irreversible type of ARD results from the same lesions as those observed in our patient.

Furthermore, regarding the recovery ability of this patient, another important factor for the recovery of cognitive abilities might be cognitive plasticity adaptation after brain damage due to excessive drinking, especially for relatively young patients as in the present case. In the future, it will be necessary to elucidate the mechanism of this plasticity, as few studies have considered this point (24).

\section{Clinical implication}

The initial symptoms in the present case included amnesia, slight disorientation, and impairment of emotional control and concentration. These symptoms are compatible with the early stage of Alzheimer's disease according to DSMIV-TR. While Alzheimer's disease is irreversible, cognitive function in the present patient showed gradual improvement after cessation of alcohol intake on hospitalization. Therefore, we finally diagnosed his illness as ARD.

The proportion of depressive patients among alcoholics is approximately 2 times higher than that among healthy population (25). In the present patient, a complication of depression was also suspected based on the lack of motivation and low GDS score in the initial evaluation. However, his mem- 
ory function had already improved at 3 weeks after abstinence when the antidepressant had not yet been administered. In addition, the topography of changes in glucose hypometabolism in our patient differed from those in patients with depression. In patients with depression, glucose hypometabolism in the prefrontal cortices is common, and these areas are associated with neuropsychological impairment $(26,27)$. The patient had no history of psychiatric disease, including depression, apathy, alcohol dependence, and drug abuse before this alcohol excessive drinking history.
Thus, we consider that depression was unlikely the main cause of cognitive decline in our patient, although it might have played a partial role in the recovery from executive dysfunction in his daily life after discharge.

In summary, we report a case of reversible ARD without thiamine deficiency that mimiced Alzheimer's disease. Detailed medical history taking is necessary for the early diagnosis and intervention of this disease. FDG-PET might also facilitate early diagnosis of this disease.

\section{References}

1. Sadock BJ, Sadock VA. In: Kaplan \& Sadock's Synopsis of Psychiatry: Behavioral Science. Clinical Psychiatry. Lippincott Williams \& Wilkins, Philadelphia, 2002.

2. Neiman J. Alcohol as a risk factor for brain damage: neurologic aspects. Alcohol Clin Exp Res 22 (Suppl. 7): 346S-351S, 1998.

3. Victor M, Adams RD, Collins GH. The Wernicke-Korsakoff syndrome and related neurologic disorders due to alcoholism and malnutrition. Davis FA, Ed. Philadelphia, 1989.

4. Reed LJ, Lasserson D, Marsden P, et al. FDG-PET findings in the Wernicke-Korsakoff syndrome. Cortex 39: 1027-1045, 2003.

5. Sechi G, Serra A. Wernicke's encephalopathy: new clinical settings and recent advances in diagnosis and management. Lancet Neurol 6: 442-455, 2007.

6. McIntosh C, Chick J. Alcohol and the nervous system. J Neurol Neurosurg Psychiatry 75 (Suppl. 3): 16-21, 2004.

7. Adams KM, Gilman S, Koeppe RA, et al. Neuropsychological deficits are correlated with frontal hypometabolism in positron emission tomography studies of older alcoholic patients. Alcohol Clin Exp Res 17: 205-210, 1993.

8. Fadda F, Rossetti ZL. Chronic ethanol consumption: from neuroadaptation to neurodegeneration. Progr Neurobiol 56: 385431, 1998.

9. Johnson-Greene D, Adams KM, Gilman S, et al. Effects of abstinence and relapse upon neuropsychological function and cerebral glucose metabolism in severe chronic alcoholism. J Clin Exp Neuropsychol 19: 378-385, 1997.

10. Lishman WA. Alcoholic dementia: a hypothesis. Lancet 1: 11841186, 1986.

11. Lishman WA. Alcohol and the brain. Brit J Psychiatry 156: 635644, 1990.

12. Wang GJ, Volkow ND, Hitzemann R, Oster ZH, Roque C, Cestaro V. Brain imaging of an alcoholic with MRI, SPECT, and PET. Am J Physiol Imaging 7: 194-198, 1992.

13. Wong DF, Maini A, Rousset OG, Brasic JR. Positron emission tomography-a tool for identifying the effects of alcohol dependence on the brain. Alcohol Res Health 27: 161-173, 2003.

14. Fiellin DA, Reid MC, O'Connor PG. Outpatient management of patients with alcohol problems. Ann Intern Med 133: 815-827, 2000.
15. Ishiai S, Koyama $Y$, Seki $K$, et al. Unilateral spatial neglect in AD: significance of line bisection performance. Neurology 55: 364-370, 2000.

16. Morino $M$, Uda $T$, Naito $K$, et al. Comparison of neuropsychological outcomes after selective amygdalohippocampectomy versus anterior temporal lobectomy. Epilepsy Behav 9: 95-100, 2006.

17. Demura S, Sato S, Tada N, Matsuzawa J, Hamasaki H. Agreement in depression determination among four self-rating depression scales applied to Japanese community-dwelling elderly. Envir Health Prev Med 11: 177-183, 2006.

18. Talairach J, Tournoux P. Co-Planar Stereotaxic Atlas of the Human Brain. Stuttgart: Thieme, 1988.

19. Chick J. Alcohol and the brain. Curr Opin Psychiatry 10: 205-210, 1997.

20. Harding A, Halliday G, Caine D, Kril J. Degeneration of anterior thalamic nuclei differentiates alcoholics with amnesia. Brain 123: 141-154, 2000.

21. Harper C. The incidence of Wernicke's encephalopathy in Australia--a neuropathological study of 131 cases. J Neurol Neurosurg Psychiatry 46: 593-598, 1983.

22. Mair WG, Warrington EK, Weiskrantz L. Memory disorder in Korsakoff's psychosis: a neuropathological and neuropsychological investigation of two cases. Brain 102: 749-783, 1979.

23. Arendt T, Bigl V, Arendt A, Tennstedt A. Loss of neurons in the nucleus basalis of Meynert in Alzheimer's disease, paralysis agitans and Korsakoff's disease. Acta Neuropathol 61: 101-108, 1983.

24. Bartsch AJ, Homola G, Biller A, et al. Manifestations of early brain recovery associated with abstinence from alcoholism. Brain 130: 36-47, 2007.

25. Sullivan LE, Fiellin DA, O'Connor PG. The prevalence and impact of alcohol problems in major depression: a systematic review. Am J Med 118: 330-341, 2005.

26. Baxter LR Jr, Schwartz JM, Phelps ME, et al. Reduction of prefrontal cortex glucose metabolism common to three types of depression. Arch Gen Psychiatry 46: 243-250, 1989.

27. Drevets WC. Functional neuroimaging studies of depression: the anatomy of melancholia. Annu Rev Med 49: 341-361, 1998.

(C) 2010 The Japanese Society of Internal Medicine

http://www.naika.or.jp/imindex.html 